\title{
Analysis of the oxypropylation process of a lignocellulosic material, almond shell, using the response surface methodology (RSM)
}

\author{
João A. Pinto ${ }^{\mathrm{a}, \mathrm{b}}$, Miguel A. Prieto ${ }^{\mathrm{c}}$, Isabel C.F.R. Ferreira ${ }^{\mathrm{a}}$, Mohamed N. Belgacem ${ }^{\mathrm{d}}$, \\ Alírio E. Rodrigues $^{\mathrm{b}}$, Maria Filomena Barreiro ${ }^{\mathrm{a}, *}$ \\ ${ }^{a}$ Centro de Investigação de Montanha (CIMO), Instituto Politécnico de Bragança, Campus de Santa Apolónia, 5300-253 Bragança, Portuga \\ ${ }^{\mathrm{b}}$ Laboratory of Separation and Reaction Engineering - Laboratory of Catalysis and Materials (LSRE-LCM), Faculty of Engineering, University of Porto, Porto, Portugal \\ ${ }^{c}$ Nutrition and Bromatology Group, Department of Analytical and Food Chemistry, Faculty of Science, University of Vigo - Ourense Campus, Ourense Spain \\ ${ }^{\mathrm{d}}$ Laboratoire de Génie des Procédés Papetiers (UMR 5518 - CNRS), Grenoble INP, Domaine Universitaire, St. Martin d'Hères, France
}

\section{A R T I C L E I N F O}

\section{Keywords:}

Almond Shell

Biomass

Biopolyols

Oxypropylation

Response surface methodology (RSM)

Optimization study

\begin{abstract}
A B S T R A C T
Developing polyols from abundant and renewable biomass resources is an important topic for polymer synthesis In this work, the Response Surface Methodology (RSM) was applied to a novel oxypropylation case study, almond shell (AS), an agroindustry lignocellulosic by-product. Mathematical models were developed to determine responses maximizing the reaction efficiency to yield polyols with specific technical requirements (polyols suitable for rigid polyurethane foams; hydroxyl index between 300 and $800 \mathrm{mgKOH} / \mathrm{g}$, and viscosity below 300 $\left.\mathrm{Pa}^{*} \mathrm{~s}\right)$. In a general way, the properties of the obtained polyols were within the range of the ones currently used commercially, reinforcing the interest to exploit lignocellulosic bio-residues for polyol synthesis. For simultaneous minimization of homopolymer content and unreacted biomass, values of $14.0 \%$ and $14.1 \%$ were achieved, respectively. This was attained using a formulation with an AS/PO ratio of $20.1 / 79.9 \mathrm{~g} / \mathrm{ml}$ and a catalyst content of $3.14 \%$, giving rise to a polyol with an hydroxyl index of $392.1 \mathrm{KOH} / \mathrm{g}$ and a viscosity of $107.4 \mathrm{~Pa}^{*} \mathrm{~s}$. Overall, the advantages of using RSM to better understand complex reactive systems and the interest to use these statistical approaches as decision-making tools was demonstrated.
\end{abstract}

\section{Introduction}

The almond is a drupe, quite produced in Portugal, in particular in the regions of Trás-os-Montes e Alto Douro, Alentejo and Algarve. In Portugal, and according to INE (Instituto Nacional de Estatística), in 2011 , the almond harvested area was 26,900 ha, and the annual production was 7700 tonnes. Thence, the production increased reaching, in $2018,39,642$ ha of cultivated area and 21,642 tonnes of annual production (INE, 2018). The world production is led by Northern America (1,872,500 tonnes), with a total contribution of $59 \%$, followed by Europe with $15 \%$ (475,513 tonnes). Spain, with a world production share of $11 \%$ (339,033 tonnes), stands out as the dominant European producer (FAOSTAT, 2020). In this context, and considering that almond fruit accounts with $80 \%(\mathrm{w} / \mathrm{w})$ of residues (hull, shell, and skin) (Esfahlan et al., 2010; Estevinho et al., 2008), approximately $2,546,321$ tonnes of these by-products can be generated, and are potentially available to develop novel applications.

Fig. 1 gives an overview of the typical operations carried out in the almond processing industry, associated primary and secondary products, as well as the generated by-products and potential applications. Some are traditional ones, but others are proposed in light of some existing studies (Holtman et al., 2015; Rosa et al., 2015; Valdés et al., 2015), and having in view the integrated valorisation of the almond processing industry value-chain. Aside from the hull, which is discarded in harvesting, shell and skin residues can be recovered as byproducts of the almond processing industry. Moreover, the shell can be used as energy source being commercialized at the cost of $0.11 € / \mathrm{kg}$ (data provided by the local producer Amendouro - Comércio e Indústria de Frutos Secos, Lda, headquartered in Alfândega da Fé, Portugal).

Face to the status of the almond cultivation and processing industry where residues can be recovered concentrated, and the fact that they achieve no, or only modest values, the proposal of new applications, namely in the field of polymeric materials are of particular interest (Rosa et al., 2015). One strategy is the use of the oxypropylation process to obtain polyols, both liquid (total oxypropylation) or biphasic (partial oxypropylation). These polyols can be used to produce polyurethanes and polyesters (Bernardini et al., 2015; Cateto et al., 2013) and onesource composites, (De Menezes et al., 2007; Rosa et al., 2014),

\footnotetext{
* Corresponding author.

E-mail address: barreiro@ipb.pt (M.F. Barreiro).
} 


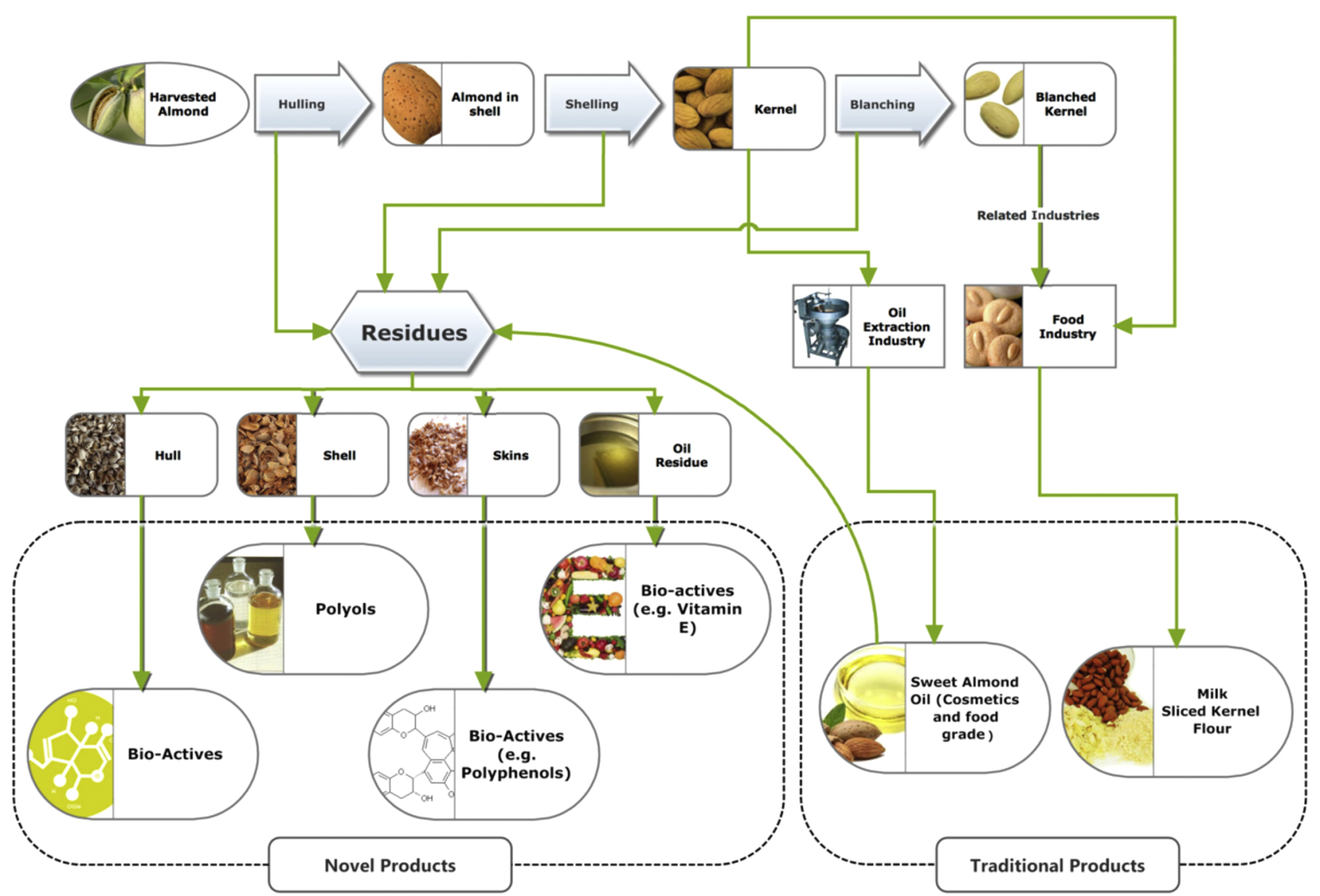

Fig. 1. Overview of the typical operations used in the almond processing industry, associated primary and secondary products, generated by-products and potential applications.

respectively.

During the past 40 years, oxypropylation has been extensively applied. Several types of biomass residues have been tested to add value to some industrial by-products. Examples include lignin (Cateto et al., 2009; Nadji et al., 2005), sodium lignosulfonates (Oliveira et al., 2015), sugar beet pulp (Pavier and Gandini, 2000), cork powder residues (Evtiouguina et al., 2002), olive stone (Matos et al., 2010), rapeseed cake (Serrano et al., 2010), and soy hulls (Rosa et al., 2015). Other biomasses, namely gambier tannin (Arbenz and Avérous, 2015), chitin and chitosan (Fernandes et al., 2007), bark (D'Souza et al., 2015), date seeds (Briones et al., 2011), and Brazilian pine fruit shell (de Rezende et al., 2018), were also studied. All these examples reported the synthesis of the polyols directly from the selected biomass, or by using a biomass pre-treatment to facilitate the impregnation of the catalyst. More recently the strategy to apply biomass fractionation was also attempted (Li et al., 2020). Another common feature is the absence of any optimization studies, only the work of de Rezende et al. (2018) provided a systematic analysis based on Principal Components Analysis (PCA), which in a context of both biomass and polyol variability is of high interest. Apart from a better understanding the oxypropylation process itself, the optimal conditions to achieve polyols with target properties can be determined, which is of industrial relevance.

The efficiency of the oxypropylation reaction varies according to several variables and operating conditions (Aniceto et al., 2012; Cateto et al., 2009). The optimal reaction conditions may not be generalized to all biomasses due to the diverse nature of their constituents (e.g. cork residues versus sugar beet pulp). Moreover, lignin, cellulose, and hemicellulose contents, and the crystalline organization, can differ, which may limit reagent's access. Another critical factor is the hydroxyl index ( $\mathrm{IOH}$, mg $\mathrm{KOH} / \mathrm{g}$ ); a biomass with a high $\mathrm{IOH}$ value requires higher amounts of reactants, namely catalyst content and more severe reaction conditions, to favour the reaction of the hydroxyls entrapped inside the biomass structure with propylene oxide. Therefore, owing to biomass variability, the selection, and optimization of the operating conditions for the oxypropylation reaction is an important topic to be studied.

In this context, one-factor-at-a-time approaches, classical methods in process optimization, can be used. Nevertheless, these simple methods cannot either accurately predict the optimal operating conditions and interactions between variables, nor provide a complete understanding of variable's patterns. One-factor-at-a-time approaches are laborious methods that, frequently, do not guarantee the reaching of the optimal conditions (Box and Hunter, 1957; Intergovernmental Panel on Climate Change, 2005). Also, carrying out experiments with all variable's combinations is impractical due to a large number of required trials (De Lean et al., 1978; Heleno et al., 2016). These limitations can be surpassed by employing the response surface methodology (RSM), which is a method specially designed to optimize responses as a function of multiple variables by requiring minimum data.

In this work, the production of biopolyols from the almond shell, a lignocellulosic agro-industrial residue, was studied, and the RSM methodology used to optimize the oxypropylation process. The chosen independent variables were the almond shell to propylene oxide ratio and the catalyst content. In terms of response variables, the hydroxyl index, homopolymer content, unreacted biomass, and viscosity were chosen. Polyol requirements were defined as the ones needed for rigid polyurethane foam preparation (IOH between 300 and $800 \mathrm{mgKOH} / \mathrm{g}$ and viscosity below $300 \mathrm{Pa*s}$ ). 


\section{Materials and methods}

\subsection{Materials}

The almond shell, designated by AS, was offered by Amendouro, Comércio e Indústria de Frutos Secos Lda (Alfandega da Fé, Portugal, http://www.amendouro.com). This material corresponds to the byproduct of the shelling process. In its original form, i.e. right after the shelling process, it is a coarse granulated material. This material was ground and after that sieved to achieve a powdered material with particle size lower than $0.5 \mathrm{~mm}$. This material was used in the characterization and oxypropylation studies. For the oxypropylation reaction, potassium hydroxide (KOH) from Panreac (Darmstdt, Germany), and propylene oxide (PO) from Aldrich (Steinheim, Germany), both p.a. grade, were use. The reactants used for AS and polyols characterization (oxypropylated samples) were of analytical grade and purchased from conventional suppliers.

\subsection{Almond shell characterization}

AS was characterized for their contents of ash and moisture, extractives (n-hexane, dichloromethane, methanol, and water), and lignocellulosic composition (hemicellulose, cellulose, and lignin), following typical methodologies used in the research group (Cateto et al., 2009; Matos et al., 2010). Next, a brief presentation is provided. All the determinations were done in duplicate.

Ash and moisture contents were determined gravimetrically. For ash, AS $(1 \mathrm{~g})$ was put in a muffle furnace $\left(550 \pm 2{ }^{\circ} \mathrm{C}, 5 \mathrm{~h}\right)$, according to Tappi 211 om-07 standard (TAPPI, 2007). For the moisture content, AS $(1 \mathrm{~g})$ was placed in an oven $\left(105 \pm 5{ }^{\circ} \mathrm{C}, 24 \mathrm{~h}\right)$, according to the ASTM 1413-76 standard (ASTM, 1984).

For the lignocellulosic composition, the respective fractions (cellulose, hemicellulose, holocellulose, and lignin) were isolated according to different extraction procedures and quantified gravimetrically. The holocellulose was extracted by the peracetic acid method $(2 \mathrm{~g}, 40 \mathrm{~min}$, $90^{\circ} \mathrm{C}$ ), under stirring and reflux, using $100 \mathrm{~mL}$ of the oxidizing medium. The sample was recovered by filtration, washed with water $\left(55^{\circ} \mathrm{C}\right)$ until neutral $\mathrm{pH}$, then with a $1: 1(\mathrm{v} / \mathrm{v})$ acetone:ethanol mixture $\left(55^{\circ} \mathrm{C}\right)$, and then dried. Hemicelluloses isolation (A and B) was performed from isolated holocellulose. Firstly, hemicellulose A was extracted with $100 \mathrm{~mL}$ of a $10 \% \mathrm{KOH}$ solution containing $14 \mathrm{mg}$ of $\mathrm{NaBH}_{4}$ using $3 \mathrm{~g}$ of holocellulose during $2 \mathrm{~h}$ at room temperature. After filtration and washing with ethanol, hemicellulose $\mathrm{B}$ was extracted by treating the remaining residue with $100 \mathrm{~mL}$ of a $24 \% \mathrm{KOH}$ solution containing $14 \mathrm{mg}$ of $\mathrm{NaBH}_{4}$. The $\alpha$-cellulose was obtained as the remaining insoluble fraction. The insoluble lignin (Klason lignin) was determined by the standardized method of Klason described in the TAPPI T222 om-08 standard, with modifications (TAPPI, 2006). Briefly, $1 \mathrm{~g}$ of AS was treated with $15 \mathrm{~mL}$ of a $72 \% \mathrm{H}_{2} \mathrm{SO}_{4}$ solution at $20^{\circ} \mathrm{C}$ under stirring for $2.5 \mathrm{~h}$. The obtained mixture was completed with water up to $300 \mathrm{~mL}$, refluxed for $2 \mathrm{~h}$, filtered and washed with boiling water until a neutral $\mathrm{pH}$ was reached.

For the extractives, a sequential extraction procedure in a soxhlet apparatus (n-hexane, dichloromethane, methanol, and water) was performed using $10 \mathrm{~g}$ of AS. The used amount of each solvent was $300 \mathrm{~mL}$ and the extraction time $6 \mathrm{~h}$. n-hexane was used to extract the fatty residues, dichloromethane to remove non-polar substances, methanol to remove the hydroxylated substances, and water to remove the most polar components. The isolated fractions were dried firstly in a rotary evaporator to concentrate, then in an oven $\left(105 \pm 5{ }^{\circ} \mathrm{C}, 2 \mathrm{~h}\right)$, and weighed.

\subsection{Oxypropylation reaction conditions and procedure}

For AS oxypropylation a $450 \mathrm{~mL}$ pressure reactor (Parr model 4560 (Illinois, USA)), equipped with a controller (model 4848) to screen pressure, temperature, and stirring, was used. This reactor enables to work with temperatures ranging up to $350^{\circ} \mathrm{C}$ and pressure up to 200 bars. For the bulk reaction, the needed amounts of AS (dried overnight in a vacuum oven at $40^{\circ} \mathrm{C}$ ), propylene oxide (PO) and catalyst (KOH) were fed into the reactor that was then closed and heated (setpoint temperature of $160^{\circ} \mathrm{C}$ ). From this initial state, pressure and temperature start to increase gradually until a maximum value, which depends on the amount of catalyst and AS/PO ratio. After reaching a maximum $\left(\mathrm{P}_{\max }\right)$, the pressure decreased rapidly, reflecting the propylene oxide consumption. When the relative pressure went to a plateau, the reactor is cooled under stirring, open, and the polyols recovered.

\subsection{Oxypropylated almond shell characterization procedures}

The polyols were characterized in what concerns the hydroxyl index (IOH), homopolymer content (HOMO), unreacted biomass (BIOMASS), and viscosity following current procedures of the research group (de Rezende et al., 2018). All the determinations were done in duplicate.

Unreacted biomass was determined gravimetrically. For that, the polyol $(1 \mathrm{~g})$ was weighted and diluted with $50 \mathrm{~mL}$ of dichloromethane. After total dissolution, the solution was filtered through a porous filter (GF/A 1.6 $\mu \mathrm{m}$ ) under vacuum, thereafter dried and weighted. The obtained residue, corresponding to the unreacted biomass, was expressed in terms of $\%(\mathrm{w} / \mathrm{w}$, biomass-basis).

Hydroxyl index (mg KOH/g) was determined according to the ASTM D4274 standard (ASTM, 2005). Briefly, the polyol (1 g) was treated with a known excess of phthalation reagent solution $(25 \mathrm{~mL})$ under reflux for $1 \mathrm{~h}$, and back-titration with $\mathrm{NaOH}(0.5 \mathrm{~N})$. The titrations were carried out with an automatic titrator TitroLine 6000 from SI Analytics (Mainz, Germany) equipped with a glass electrode.

For homopolymer quantification, the extraction from the produced polyol was performed with n-hexane under reflux (two cycles) according to the methodology proposed by Pavier and Gandini (2000). The recovered fractions, homopolymer and oxypropylated AS, were vacuum-dried in an oven until constant weight was reached. The results were expressed in $\%(w / w)$.

Viscosity was determined in a Analogic Rheometer Malvern CVO 100 (Westborough, MA, USA) at $20^{\circ} \mathrm{C}$, under stress-controlled mode operation $\left(40 \mathrm{~mm}\right.$ diameter, $4^{\circ}$ top-angle cone plate with a gap of $150 \mu \mathrm{m})$. Data was acquired in a shear stress range of 15-3500 Pa using 50 points.

\subsection{Response surface methodology (RSM)}

RSM is used to model and analyse problems where responses are influenced by several variables. In oxypropylation optimization, the independent variables were the catalyst content (Cat, $\left.X_{1}\right)$ in $\%(w / w$, AS-basis)), and the AS to propylene oxide (PO) ratio (AS/PO ratio, $X_{2}$ ) in $\mathrm{g} / \mathrm{mL}$. The responses (dependent variables) were the hydroxyl index ( $\mathrm{IOH}, Y_{1}$ ), expressed in $\mathrm{mg} \mathrm{KOH} / \mathrm{g}$; the homopolymer content (HOMO, $Y_{2}$ ) expressed in \% $(\mathrm{w} / \mathrm{w})$; unreacted biomass (BIOMASS, $Y_{3}$ ), expressed in $\%\left(\mathrm{w} / \mathrm{w}\right.$, polyol-basis) and viscosity (Viscosity, $\left.Y_{4}\right)$, expressed in $\mathrm{Pa} * \mathrm{~s}\left(20^{\circ} \mathrm{C}\right)$.

\subsubsection{Experimental design}

The combined effects of Cat $\left(X_{1}\right)$ and AS/PO ratio $\left(X_{2}\right)$ variables on AS oxypropylation was done using a full factorial design (2 replicates per condition). The structure of a full factorial design needs, per factor, to address all three-values combinations (minimum, mean and maximum). The number of experiments for $k$ factors is $n=2^{k}$, where $n$ is the number of experiments. Experimental runs were randomized to minimize unpredicted variability effects in the observed responses. The variables were coded following the next equation:

$X=\left(x_{a}-x_{0}\right) / \Delta x$

where $X$ is the coded value for the variables $A S / P O$ and Cat, $x_{a}$ the 
corresponding actual value, $x_{0}$ the actual value in the centre of the domain, and $\Delta x$ the increment of $x_{a}$ leading to a variation of 1 unit in $X$. The conditions of the studied independent variables are reported in Table A1 (supplemental material), which summarizes the procedure for variables encoding and applied experimental conditions.

\subsubsection{Mathematical model}

The RSM model was fitted through least-squares regression using the next second-order polynomial equation:

$Y=b_{0}+\sum_{i=1}^{n} b_{i} X_{i}+\sum_{\substack{i=1 \\ j>i}}^{n-1} \sum_{j=2}^{n} b_{i j} X_{i} X_{j}+\sum_{i=1}^{n} b_{i i} X_{i}^{2}$

where $Y$ is the response (dependent variable), $X_{i}$ and $X_{j}$ the independent variables, $b_{o}$ the constant coefficient, $b_{i}$ the linear effect coefficient, $b_{i j}$ the interaction effect coefficient, $b_{i i}$ the quadratic effect coefficient, and $n$ the number of variables.

\subsection{Numerical methods and statistical analysis}

All fitting procedures, coefficient estimates, and statistical calculations were performed on Microsoft Excel. The fitting of the experimental results to the defined equations and subsequent statistical analysis was carried in four phases: (a) Coefficients determination: Parametric estimates were determined by minimization of the sum of the quadratic differences between observed and model-predicted values. For that, the nonlinear least-square (quasi-Newton) Microsoft Excel macro Solver (Kemmer and Keller, 2010) was used. Among the advantages, it enables a quick hypothesis testing and evaluation of effects (Prieto and Vázquez, 2014); (b) Coefficients significance: The determination of the parametric confidence intervals was done with 'SolverAid' (Albuquerque et al., 2016), and the model was simplified by dropping the non-significant terms for $p$-value $(p)>0.05$; (c) Model consistency: The Fisher $F$ test $(<=0.05)$ was used to determine the adequacy of the models to describe the observed data (Shi and Tsai, 2002); and (d) Other statistical assessment criteria: Model uniformity was rechecked with next criteria: i) The 'SolverStat' macro (Comuzzi et al., 2003), which enables the assessment of parameter and model prediction uncertainties; ii) $\mathrm{R}^{2}$, the percentage variability of the dependent variable explained by the model; iii) $R_{a d j}^{2}$, adjusted coefficients of multiple determination, a correction to $R^{2}$ considering the number of variables of the model; iv) Bias and accuracy parameters, calculated to evaluate the fittings, namely MSE (Mean Squared Error), RMSE (Root Mean Square of Errors) (RMSE), and MAPE (Mean Absolute Percentage Error; v) The Durbin-Watson coefficient (DW), used to check autocorrelation between model residuals; and vi) ANOVA (analysis of variance) to evaluate the descriptive power of the variables.

\section{Results and discussion}

According to literature, oxypropylation conditions vary with biomass type, which evidences the interest to use statistical tools to optimize the conditions favouring the process. Therefore, a statistical RSM design was applied to examine the best experimental conditions to produce polyols from AS having in view their use in the preparation of rigid polyurethane foams (polyol requirements: IOH between 300 and 800 and viscosity below $300 \mathrm{~Pa}^{*} \mathrm{~s}$ ).

\subsection{Almond shell characterization}

The AS used in this study is a lignocellulosic material with a moisture content of $6 \%(\mathrm{w} / \mathrm{w})$. A complete description of the lignocellulosic composition, extractives (n-hexane, dichloromethane, methanol and water), and ashes ( $\mathrm{w} / \mathrm{w}$, dry basis) is given in Table 1 . The obtained results agree with other ones reported in published works
Table 1

Chemical composition (dry-basis) of the almond shell used in this study.

\begin{tabular}{ll}
\hline $\begin{array}{l}\text { ALMOND SHELL } \\
\text { COMPONENT }\end{array}$ & $\begin{array}{l}\text { AMOUNT } \\
\text { (\%, W/W) }\end{array}$ \\
\hline $\begin{array}{ll}\text { Ash content } \\
\text { Lignocellulosic components } \\
\text { Cellulose }\end{array}$ & 2.53 \\
Hemicelluloses & 34.39 \\
Holocellulose & 13.96 \\
Insoluble Lignin & 48.34 \\
Soluble Lignin & 39.26 \\
Extractables & 0.66 \\
n-Hexane & \\
Dichloromethane & 1.01 \\
Methanol & 0.18 \\
Water & 4.00 \\
\hline
\end{tabular}

(Aktas et al., 2015). The low ash content is within the range typical for lignocellulosic materials (around 3\%), except for rice straw that presents a quite high ash content (17.5\%) (Pandey et al., 2000). One particularity of the AS is the high lignin content (reaching 40\%), comparatively with other lignocellulosic materials, such as sugar cane bagasse (25\%) (Pandey et al., 2000), olive stone (26\%) (Matos et al., 2010), and almond hulls (12\%) (Holtman et al., 2015). Another feature is the high content of extractives that reached a value of $9.2 \%$ accounting with $1 \%$ of fats (based on the n-hexane extractives), less than $1 \%$ of non-polar substances (based on the dichloromethane extractives), and equivalent values (4\%) of OH-bearing substances and more polar substances, based respectively in methanol and water extractives.

\subsection{Variables selection and centre of the experimental design}

In the context of the oxypropylation reaction, previous studies indicated $\mathrm{KOH}$ as the most used catalyst. Namely, in the work of Pavier and Gandini (2000), a comparative study using different catalysts, the efficacy of $\mathrm{KOH}$ was corroborated. Moreover, $\mathrm{KOH}$ is widely used industrially in polyether-polyols synthesis. In what concerns temperature, setpoint temperature is usually lower for high lignin content materials (e.g. pure lignin materials use $160-180^{\circ} \mathrm{C}$ ) (Cateto et al., 2009; Nadji et al., 2005) and/or high hemicellulose content (e.g. date seeds, with a hemicellulose content of $55 \%$, and rapeseed cake with $44 \%$ also use $160{ }^{\circ} \mathrm{C}$ ) (Briones et al., 2011; Serrano et al., 2010). Thus, based on the determined AS lignocellulosic composition, combined with the interest to use moderate temperatures to promote energetically competitive processes, $160{ }^{\circ} \mathrm{C}$ was chosen in this study. In addition to the previous assumptions, one global objective in biomass valorization is to use/ convert the highest amount possible.

Following the previous considerations that fixed $\mathrm{KOH}$ as the catalyst, and $160^{\circ} \mathrm{C}$ as the chosen setpoint temperature, the selected relevant independent variables were the catalyst content ( $\mathrm{Cat}, \mathrm{X}_{1}$ ), expressed as $\%(\mathrm{w} / \mathrm{w}$, AS based), and the AS to propylene oxide ratio (AS/ $P O$ ratio, $X_{2}$ ) expressed in $\mathrm{g} / \mathrm{mL}$. Preliminary experimental trials have shown that lower Cat (inferior to $5 \%$ ) lead to very viscous polyols, particularly in the case of using high $A S / P O$ ratios. Therefore, the selected ranges for process optimization were 5,10 , and $15 \%$, for Cat, and 10/90, 20/80 and 30/70 (w/v), for AS/PO ratio.

\subsection{Establishment of mathematical models and validation}

The design of the RSM experiment took into account the preliminary experimental results, which led to the definition of Cat and AS/PO ratio as the relevant variables. A full factorial design with three levels was used, and the best fitting solution was predicted using a second-order polynomial model, by non-linear least-squares minimization. Results of the response surface experimental plan for the optimization of Cat $\left(X_{1}\right)$ 
Table 2

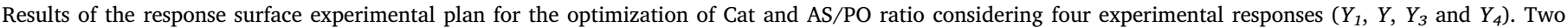
replicates $\left(r_{1-2}\right)$ were considered for each condition and each response.

VARIABLES

\begin{tabular}{ll}
\hline$X_{1}:$ Cat & $\begin{array}{l}X_{2}: A S / P O \\
(\% g / m L)\end{array}$ \\
$-1(5)$ & $-1(10 / 90)$ \\
$-1(5)$ & $0(20 / 80)$ \\
$-1(5)$ & $1(30 / 70)$ \\
$0(10)$ & $-1(10 / 90)$ \\
$0(10)$ & $0(20 / 80)$ \\
$0(10)$ & $1(30 / 70)$ \\
$1(15)$ & $-1(10 / 90)$ \\
$1(15)$ & $0(20 / 80)$ \\
$1(15)$ & $1(30 / 70)$
\end{tabular}

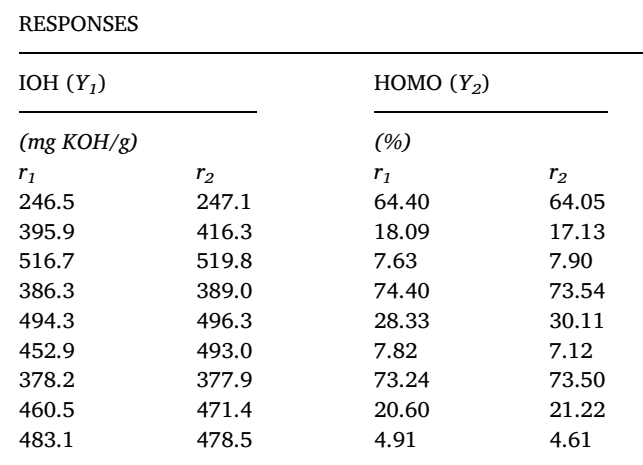

\begin{tabular}{ll} 
BIOMASS $\left(Y_{3}\right)$ & \\
\hline$(\%)$ & \\
$r_{1}$ & $r_{2}$ \\
11.0 & 9.0 \\
7.0 & 10.5 \\
28.7 & 32.7 \\
5.0 & 6.0 \\
9.0 & 10.0 \\
31.0 & 35.0 \\
6.0 & 5.0 \\
22.0 & 26.5 \\
37.3 & 42.7
\end{tabular}

\begin{tabular}{ll} 
Viscosity $\left(Y_{4}\right)$ & \\
\hline$\left(\mathrm{Pa}^{*} \mathrm{~s}\right)$ & \\
$r_{1}$ & $r_{2}$ \\
3.5 & 3.9 \\
77.1 & 67.6 \\
247.1 & 233.5 \\
0.5 & 0.5 \\
11.7 & 7.3 \\
73.5 & 61.0 \\
0.5 & 0.6 \\
23.9 & 24.6 \\
134.2 & 110.1 \\
\hline
\end{tabular}

and AS/PO ratio $\left(X_{2}\right)$ considering the four defined experimental responses (IOH, HOMO, BIOMASS, and viscosity; Y1- Y4) are shown in Table 2 . The criteria response results $\left(Y_{1^{-}} Y_{4}\right)$ were obtained by running 18 trials (9 genuine combination conditions with 2 replicas per condition) according to the defined RSM design (Table A1).

\subsubsection{Theoretical response surface model}

Estimated coefficient values of Eq. [2], parametric intervals, and numerical statistical criteria, for responses $Y_{1}-Y_{4}$, are shown in Table 3. The model gave, for all the examined cases, statistically significant coefficients ( $p$-values lower than 0.05 at $95 \%$ confidence level). The following second-order polynomial equations were derived from Eq. [2]:

$\operatorname{IOH}\left(\mathrm{Y}_{1}\right): Y_{1}=479.7+25.6 x_{1}+76.5 x_{2}-35.9 x_{1}^{2}-41.7 x_{2}^{2}-42.2 x_{1} x_{2}$

$\operatorname{HOMO}\left(Y_{2}\right): Y_{2}=26.2+1.5 x_{1}-31.9 x_{2}-5.5 x_{1}^{2}-16.1 x_{2}^{2}-3.1 x_{1} x_{2}$

$\operatorname{BIOMASS}\left(Y_{3}\right): Y_{3}=11.6+3.4 x_{1}+13.8 x_{2}+3.9 x_{1}^{2}+6.6 x_{2}^{2}+3.5 x_{1} x_{2}$
$\operatorname{Viscosity}\left(Y_{4}\right): Y_{4}=14.2-28.2 x_{1}+60.9 x_{2}+31.8 x_{1}^{2}+46.8 x_{2}^{2}-28.7 x_{1} x_{2}$

Although the gotten model coefficients are empirical, they can be used to predict the results of untested operational conditions (Heleno et al., 2016), namely the sign can be used to perceive the response performance. If it is positive, the effect in the response is higher at the high level; if the factor is negative, the effect in the response is lower at the high level. The higher the absolute value is, the more relevant the variable's weight is.

Fig. 2 shows the criteria response results $\left(Y_{1}-Y_{4}\right)$. Each figure is divided into three subsections (A, B and $\mathrm{C}$ ). The subsection A shows the three-dimensional response surface plots for the response criteria as a function of Cat $\left(X_{1}\right)$ and $A S / P O$ ratio $\left(X_{2}\right)$ variables, as predicted by the second-order polynomial equations (Eqs. (3), (4) (5) and (6)). Points (O) represent the obtained experimental results (numerical values in Table 2). Estimated parametric values are shown in Table 3. In subsection $\mathrm{B}$, the fitting (solid line) of the two-dimensional representations of Eqs. [3]-[6] to the experimental points ( $\square$ minimum, $\triangle$ medium and $\diamond$ maximum variable values), is presented considering the combined effect of Cat and AS/PO ratio variables. In subsection $\mathrm{C}$ the

Table 3

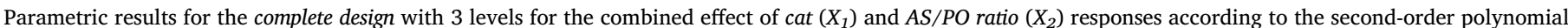

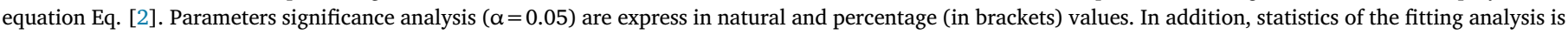
presented.

\begin{tabular}{|c|c|c|c|c|c|c|c|c|c|c|c|c|c|}
\hline & & \multicolumn{12}{|c|}{ RESPONSES } \\
\hline & & \multicolumn{3}{|l|}{$\mathrm{IOH}\left(\mathrm{Y}_{1}\right)$} & \multicolumn{3}{|c|}{$\mathrm{HOMO}\left(\mathrm{Y}_{2}\right)$} & \multicolumn{3}{|c|}{ BIOMASS $\left(\mathrm{Y}_{3}\right)$} & \multicolumn{3}{|c|}{ Viscosity $\left(\mathrm{Y}_{4}\right)$} \\
\hline & & \multicolumn{3}{|c|}{$(m g \mathrm{KOH} / g)$} & \multicolumn{3}{|l|}{$(\%)$} & \multicolumn{3}{|l|}{$(\%)$} & \multicolumn{3}{|l|}{$(P a * s)$} \\
\hline \multicolumn{14}{|c|}{ Fitting coefficients obtained } \\
\hline Intercept & $b_{o}$ & 479.76 & \pm 28.60 & $(6 \%)$ & 26.21 & \pm 2.20 & $(8 \%)$ & 11.59 & \pm 4.05 & $(35 \%)$ & 14.16 & \pm 2.93 & $(21 \%)$ \\
\hline \multirow[t]{2}{*}{ Linear effect } & $b_{1}$ & 25.602 & \pm 15.67 & $(61 \%)$ & 1.57 & \pm 1.20 & $(76 \%)$ & 3.38 & \pm 2.22 & $(66 \%)$ & -28.24 & \pm 18.78 & $(67 \%)$ \\
\hline & $b_{2}$ & 76.582 & \pm 15.67 & $(20 \%)$ & -31.93 & \pm 1.20 & $(4 \%)$ & 13.78 & \pm 2.22 & $(16 \%)$ & 60.99 & \pm 18.78 & $(31 \%)$ \\
\hline \multirow[t]{2}{*}{ Quadratic effect } & $b_{11}$ & -35.95 & \pm 27.13 & $(75 \%)$ & -5.45 & \pm 2.08 & $(38 \%)$ & 3.87 & \pm 3.84 & $(99 \%)$ & 31.83 & \pm 12.93 & $(41 \%)$ \\
\hline & $b_{22}$ & -41.72 & \pm 27.13 & $(65 \%)$ & 16.01 & \pm 2.08 & $(13 \%)$ & 6.62 & \pm 3.84 & $(58 \%)$ & 46.83 & \pm 12.93 & $(28 \%)$ \\
\hline Interactive effect & $b_{12}$ & -42.18 & \pm 19.19 & $(45 \%)$ & -3.04 & \pm 1.47 & $(49 \%)$ & 3.45 & \pm 2.72 & $(79 \%)$ & -28.75 & \pm 12.93 & $(45 \%)$ \\
\hline \multicolumn{14}{|c|}{ Statistical information of the fitting analysis } \\
\hline Obs & & 18 & & & 18 & & & 18 & & & 18 & & \\
\hline$d f$ & & 12 & & & 12 & & & 12 & & & 12 & & \\
\hline$R^{2}$ & & 0.9192 & & & 0.9960 & & & 0.9496 & & & 0.9081 & & \\
\hline$R^{2} a d j$ & & 0.8855 & & & 0.9943 & & & 0.9286 & & & 0.8698 & & \\
\hline$M E C$ & & 9484 & & & 1128 & & & 206 & & & 8483 & & \\
\hline RMSE & & 97.4 & & & 33.6 & & & 14.3 & & & 92.1 & & \\
\hline MAPE & & 4.4 & & & 12.5 & & & 18.5 & & & 1224.7 & & \\
\hline$D W$ & & 1.6 & & & 3.1 & & & 3.4 & & & 1.5 & & \\
\hline
\end{tabular}

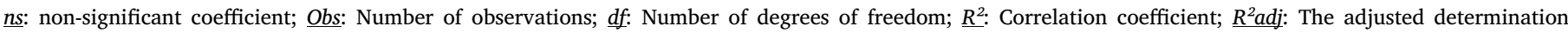

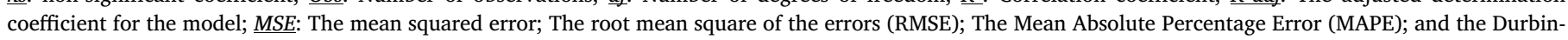
Watson (DW) statistics. 

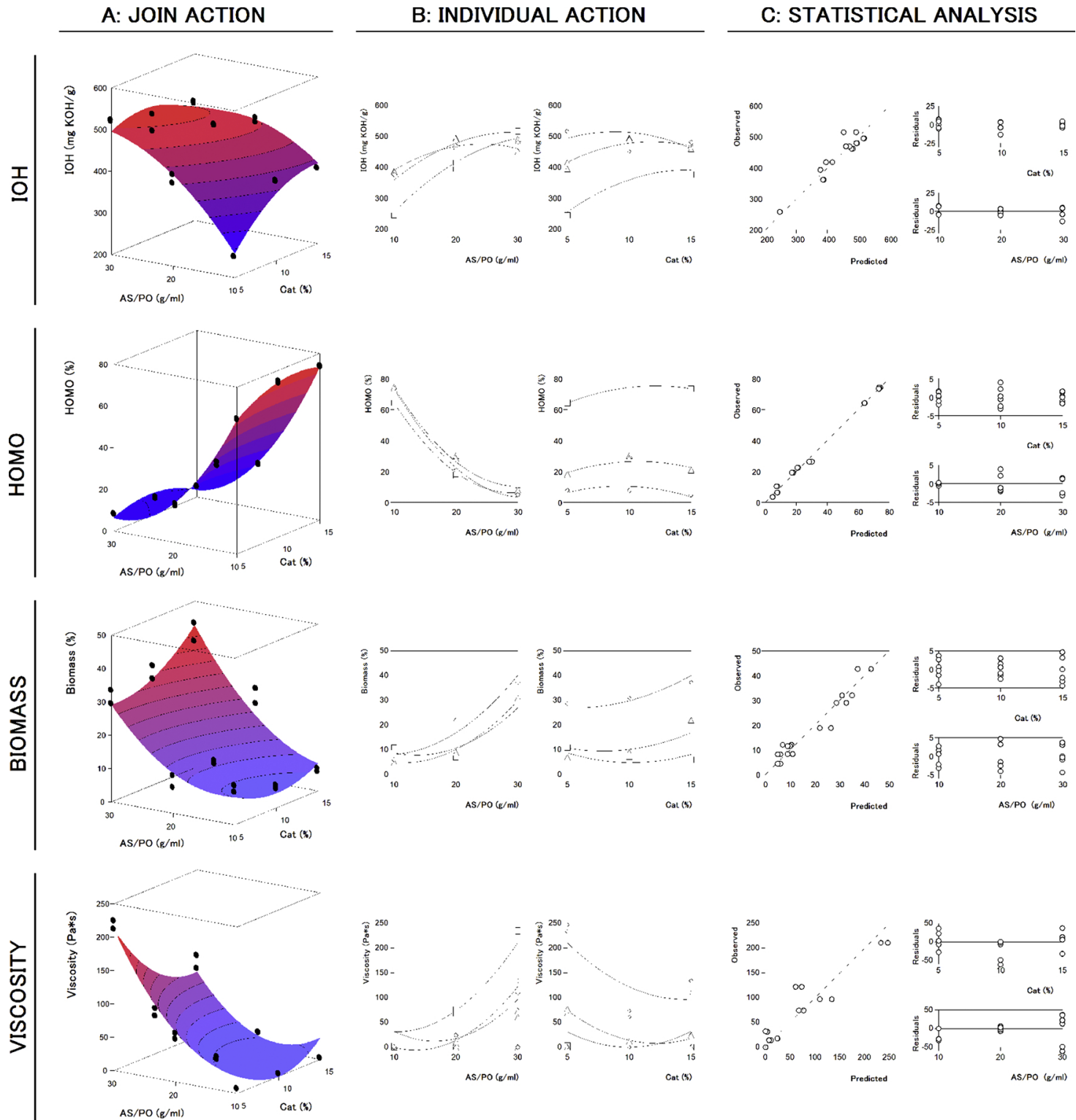

Fig. 2. Graphical results in terms of viscosity, unreacted biomass, homopolymer content and hydroxyl index. Part A: Shows the joint graphical 3D analysis as a function of each considered variable. Each of the net surfaces represents the theoretical three-dimensional response surface predicted by the second-order polynomials (Eqs. [3], [4], [5] and [6]) for all format values $\left(Y_{1}, Y_{2}, Y_{3}\right.$ and $Y_{4}$, respectively). The binary action between variables considers that the excluded variable is positioned at the centre of the experimental domain $(\mathrm{Cat}=10 \% ; A S / P O=20 / 80)$. The statistical design and results are described in Table 2. Estimated parametric values are shown in Table 3 - Part B: Two-dimensional representation of Eqs. (3), (4), (5) and (6) fitting (solid line) to the experimental results ( $\square$ minimum, $\diamond$ medium and $\triangle$ maximum variable values) of $C a t\left(X_{1}\right)$ and $A S / P O\left(X_{2}\right)$ combined effect. Part $C$ : Illustration of the goodness of fit following two essential graphical statistic criteria: (1) the ability to simulate response changes between predicted and observed values; (2) the distribution of the residuals as a function of the individual variables.

residual distribution as a function of the defined variables (Cat and AS/ $P O$ ratio) is shown illustrating the capacity to predict the results.

\subsubsection{Statistical and experimental verification of predictive models}

The multivariable characterization of the second-order polynomial model is exceptionally robust, minimizing the effects of random and systematic errors. Optimal and efficient data analysis should involve the simultaneous description of all curves, rather than fitting each one individually (Curran et al., 2013; De Lean et al., 1978). Namely, the simultaneous curve-fitting reduces the number of parameters needed to analyse the response, becoming more informative, i.e. providing better parameter's estimation, and reducing their confidence interval (Murado and Prieto, 2013). Once the action modes are mathematically identified, if experimental curves do not span the full range, and some fail to provide information about one or more parameters, the multivariable application can be a solution to describe all the areas. By standardizing the response, the obtained results are less dependent on the experimental conditions, which is a common problem of the efficacy of the response factors analysis.

The statistic lack of fit, used to test the adequacy of the obtained models, demonstrated that significant improvement was reached by the exclusion of the statistically non-significant effects (Table 3). This was corroborated by the determined high $R^{2}$ and $R_{\text {adj }}^{2}$ values, which indicate the percentage of variability explained by the model (Table 3 ). Additionally, Fig. 2 (subsection $\mathrm{C}$ ) shows that residuals distribution is always randomly scattered around zero, with no grouped data and autocorrelations. This means that the models are feasible and can be subsequently applied in the prediction and optimization stages, 
indicating a good agreement between experimental and predicted values.

Table A2 (supplementary material) shows the analysis of variance (ANOVA) for the regression equation. The linear and quadratic terms were highly significant $(p<0.01)$. The lack of fit, to check model acceptability, showed to be not significant $(p>0.05)$, indicating a good fit of the experimental data.

\subsection{Analysis of the oxypropylation process}

The obtained polyols were brown liquids with variable viscosity presenting no solid deposits, even when unreacted biomass was present (Figure A1, supplemental material).

After the mathematical models were established, and validated statistically for the accessed responses $\left(Y_{1}-Y_{4}\right)$, as a function of the studied variables $\left(X_{1}\right.$ and $\left.X_{2}\right)$, the system can be scrutinised more comprehensively, i.e. comparatively with an independent analysis of the experimental results.

The conditions maximizing the oxypropylation process can be evaluated using a multivariable system procedure by dividing the responses into two groups:

R1)The restricted responses $\left(Y_{1}\right.$ and $\left.Y_{4}\right)$, which are those with fixed values within a marginal range $\left(Y_{1}, \mathrm{IOH}\right.$, from 300 to 800 , and $Y_{4}$, viscosity, below 300);

R2)The target responses ( $Y_{2}$ and $Y_{3}$ ), which represent the focus of the optimization process. For these variables, $Y_{2}$ (HOMO) and $Y_{3}$ (BIOMASS), the lower the values, the more effective is the oxypropylation process. Low unreacted biomass means that a high conversion to liquid polyol was achieved. A low homopolymer content means that propylene oxide mostly reacted with the biomass, and a low level was involved in secondary reactions.

Two optimization approaches were performed:

A1)The optimization of $X_{1}$ and $X_{2}$ variables (process variables) minimizing $Y_{2}$ and $Y_{3}$ (responses), individually and conjointly, by respecting the restricted responses $\left(Y_{1}\right.$ and $\left.Y_{4}\right)$ within the marginal values, by solving the regression Eqs. (3),(4),(5) and (6), via equating the partial derivatives to zero and decoding the code value to its natural value, or by using a simplex procedure;

A2)The minimization of the responses $Y_{2}$ and $Y_{3}$ by rearranging the Eqs. [3], [4], [5] and [6] in a multivariable form to produce all the response profiles jointly as a function of the variables, and therefore, understand the oxypropylation process globally.

From the approach A1, either by equating the partial derivatives to zero or by applying the simplex procedure, the results (Fig. 3) pointed out that the optimized conditions minimizing $Y_{2}$ and $Y_{3}$ responses (keeping $Y_{1}$ and $Y_{4}$ within the established marginal values) are as follows:

- When Y2 (HOMO) was minimized, the predicted minimum value was $0.03 \%$. The corresponding values for the other responses were $344.3 \mathrm{mg} \mathrm{KOH} / \mathrm{g}$ for the IOH (Y1), 20.35\% for BIOMASS (Y3) and $200.5 \mathrm{~Pa}$ *s for viscosity (Y4). The optimized conditions indicated for $\mathrm{X} 1$ (catalyst content) and X2 (AS/PO ratio) $1.57 \%$ and $22.5 / 77.5 \mathrm{~g} /$ $\mathrm{ml}$, respectively;

- When Y3 (BIOMASS) was minimized the minimum predicted value was $4.20 \%$, with the other responses assuming the following values: $347.8 \mathrm{mg} \mathrm{KOH} / \mathrm{g}(\mathrm{Y} 1, \mathrm{IOH}), 77.35 \%$ for (Y2, HOMO), and $2.9 \mathrm{~Pa} * \mathrm{~s}$ (Y4, viscosity). The optimized variable conditions were $10.57 \%$ for $\mathrm{X} 1$ (catalyst content) and 8.5/91.5 g/ml for X2 (AS/PO ratio);

- When Y2 (HOMO) and Y3 (BIOMASS) were minimized co-jointly, the minimum predicted values were $14.0 \%$ and $14.1 \%$, respectively. The corresponding values for the other responses were $392.1 \mathrm{mg}$ $\mathrm{KOH} / \mathrm{g}(\mathrm{Y} 1, \mathrm{IOH})$ and 107.4 Pa*s (Y4, viscosity). For the optimized variables, values of $3.14 \%$ (X1, catalyst content) and 20.1/79.9 g/ml (X2, AS/PO ratio) were achieved.
This optimization approach (A1) corresponds to the usual procedure in RSM design, and no further conclusions are stressed-out. However, in the case under study (oxypropylation process), once the models were established (Eqs. [3]-[6]), the patterns of the restricted responses $\left(Y_{1}\right.$ and $Y_{4}$ ), and variables $\left(X_{1}\right.$ and $\left.X_{2}\right)$, were found for all possible minimization solutions of the target responses $\left(Y_{2}\right.$ and $\left.Y_{3}\right)$, providing a global interpretation of the process. This optimization is performed according to the previously described A2 approach and is presented in Fig. 4. Also, in Fig. 4, the results of the A1 optimization approach, namely the independent minimum values of $Y_{2}$ and $Y_{3}$, and the global minimum for both variables, is represented by dot points ( $)$ alongside the line of the minimization solutions of the target responses $\left(Y_{2}\right.$ and $Y_{3}$ ). Both optimization approaches (A1 and A2) meet in the same conclusions, but the second one is much more informative. The following conclusions can be stressed out from the profiles represented in Fig. 4, for the variables and responses involved in the oxypropylation process:

- The variable X1 (catalyst content) shows a linear relationship, which decreases as the minimization solutions of the Y2 (HOMO) and Y3 (BIOMASS) responses decrease. Low homopolymer content and unreacted biomass are favoured when low catalyst contents are used. Appositively, high homopolymer content and unreacted biomass are achieved for high catalyst contents.

- The variable X2 (AS/PO ratio) shows a linear dependency that increases as the minimization solutions of the Y2 (HOMO) and Y3 (BIOMASS) responses decrease. Low AS/PO ratios give rise to low unreacted biomass and high homopolymer content. As the AS/PO increases, unreacted biomass increases and homopolymer content decreases.

- The response Y4 (viscosity) shows a decreasing hyperbolic relationship as the minimization solutions of the Y2 (HOMO) and Y3 (BIOMASS) decrease. Low viscosity polyols are formed when a high amount of homopolymer is found in the mixture, together with a low unreacted biomass content. Moreover, for low enough unreacted biomass (i.e. full conversion of biomass) the viscosity is almost invariant with homopolymer content increase.

- The response Y1 (IOH) shows a bell relationship as the minimization solutions of the Y2 (HOMO) and Y3 (BIOMASS) responses increasing from the minimum solution of Y2 (HOMO), thus reaching a maximum value close the joint minimization solution of Y2 (HOMO) and Y3 (BIOMASS), and then, decreases again towards the minimum solution of $Y_{3}$ (BIOMASS).

\section{Conclusions}

In this work, and by using a novel case study (almond shell), the advantages of using RSM was demonstrated in the analysis of the oxypropylation process. To the best of our knowledge this is the first work dealing with this approach. Through this analysis the effect of typical process variables (AS/PO ratio and catalyst content) on the defined response variables (hydroxyl index, homopolymer content, unreacted biomass, and viscosity) was systematized. Moreover, the use of restricted responses, having in view the achievement of target technical requirements, here represented as the polyol requirements needed for the synthesis of rigid polyurethane foams, was demonstrated. When both homopolymer content and unreacted biomass were minimized, which is essential having in view high biomass conversion and the minimization of secondary reactions, the achieved values were $14.0 \%$ and $14.1 \%$, respectively. The other technical polyol parameters comprised an $\mathrm{IOH}$ of $392.1 \mathrm{KOH} / \mathrm{g}$ and a viscosity of 107.4 Pa*s. This was achieved by using an AS/PO ratio of $20.1 / 79.9 \mathrm{~g} / \mathrm{ml}$ and a catalyst content of $3.14 \%$, formulation comprising a modest content of biomass. The incorporation of a high amount of biomass is desirable from a biobased product perspective. Nevertheless, the use of higher AS/PO ratios favour the increase of unreacted biomass in the final polyol 
J.A. Pinto, et al.

Industrial Crops \& Products 153 (2020) 112542

$\mathrm{Y}_{1}: \mathrm{IOH}(\mathrm{mg} \mathrm{KOH} / \mathrm{g})$
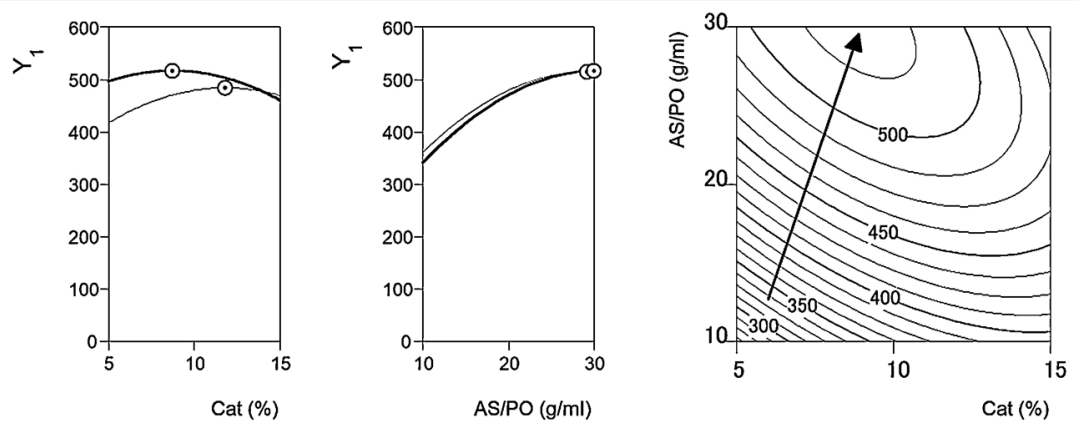

$\mathrm{Y}_{2}:$ HOMO (\%)
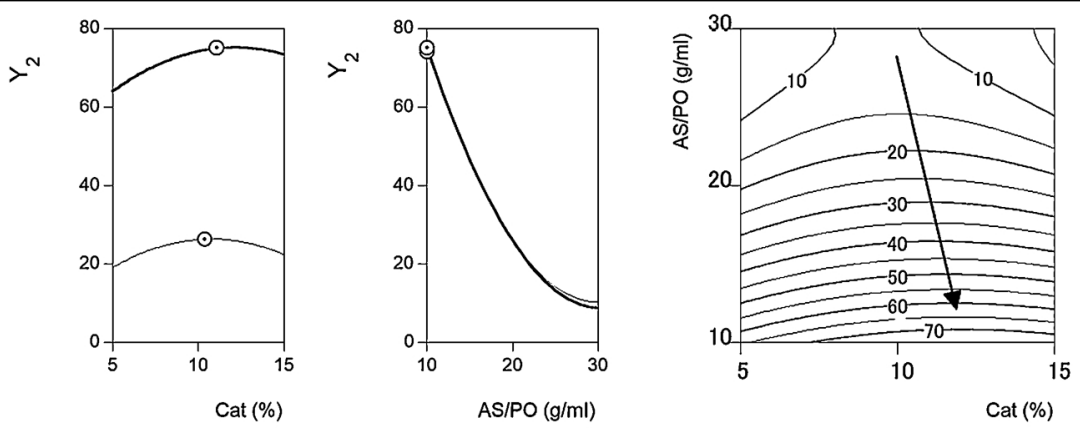

$Y_{3}$ : Biomass (\%)
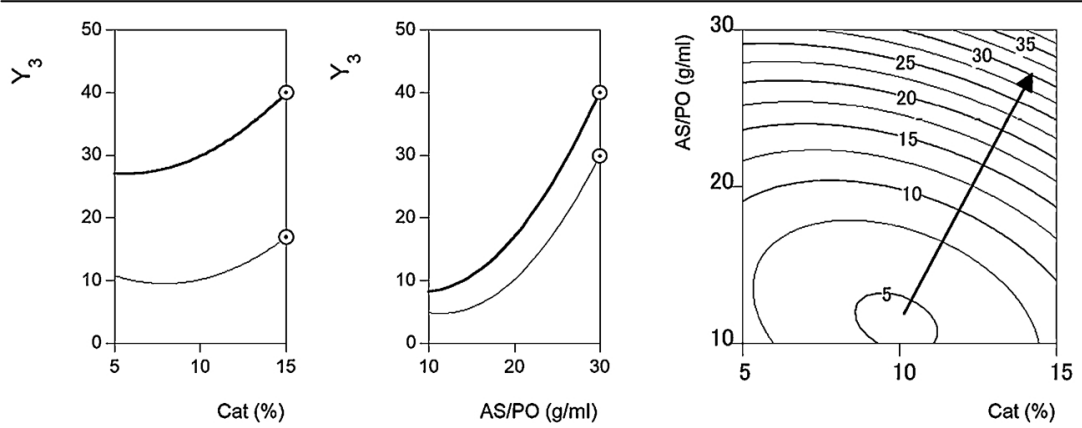

$\mathrm{Y}_{4}$ : Viscosity ( $\left.\mathrm{Pa}^{*} \mathrm{~s}\right)$
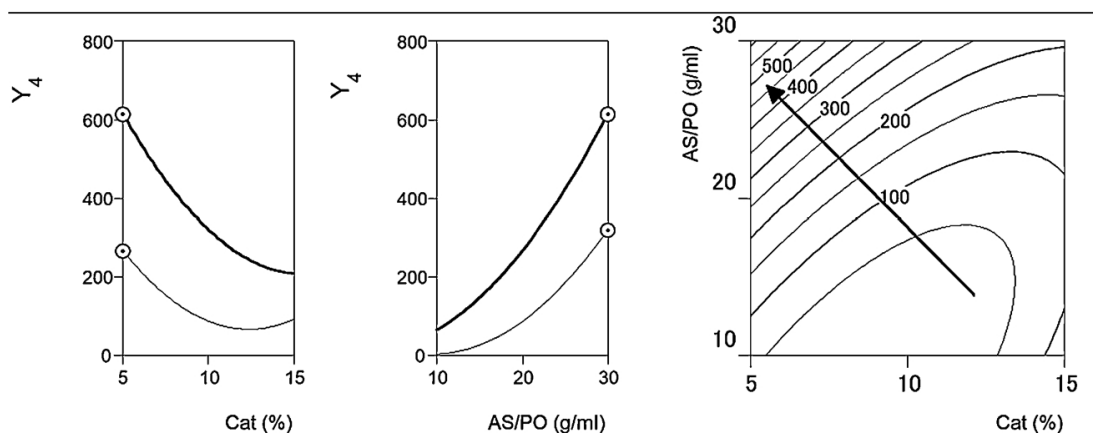

8 


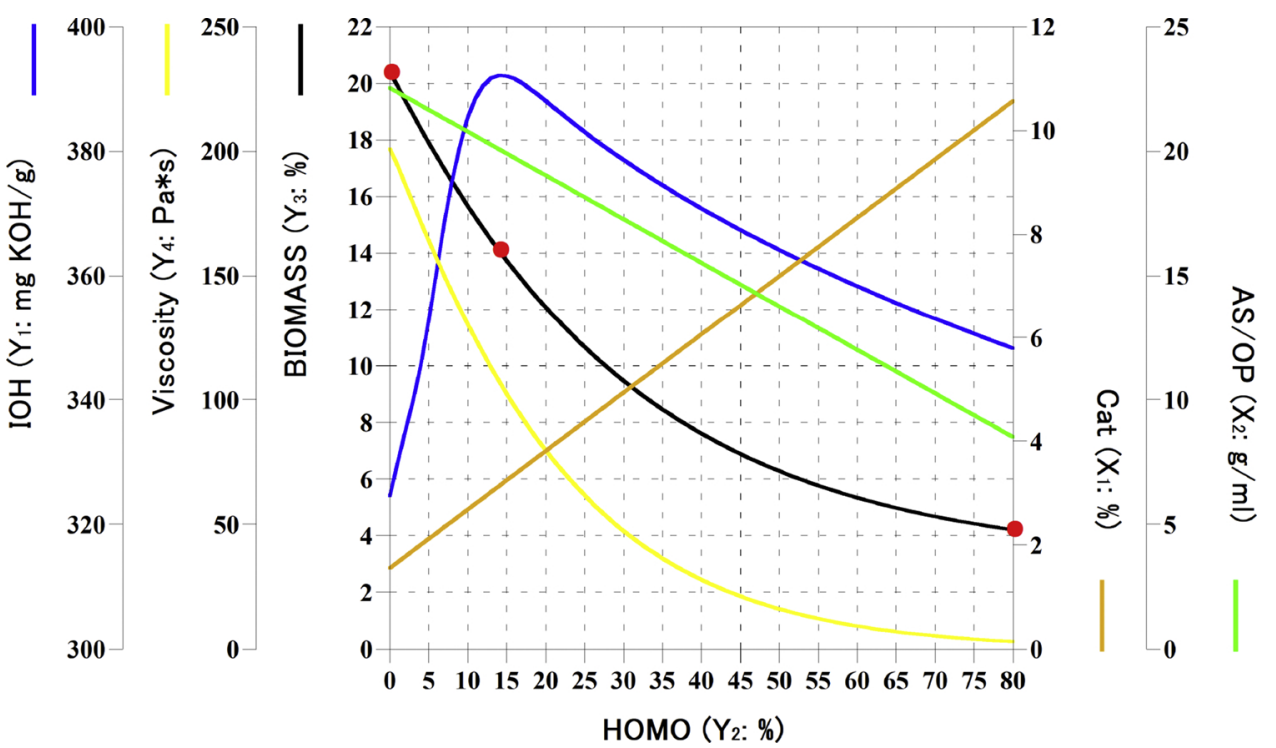

Fig. 4. Global interpretation of the oxypropylation process obtained from the multivariable system described in the A2 approach using the models of Eqs. [3] to [6]. The patterns of the restricted responses $\left(Y_{1}\right.$ and $\left.Y_{4}\right)$, and variables $\left(\mathrm{X}_{1}\right.$ and $\left.\mathrm{X}_{2}\right)$ as a function of all possible minimization solutions of target responses $\left(\mathrm{Y}_{2}\right.$ and $\mathrm{Y}_{3}$ ), are shown. The dots $(\bullet)$ presented along the lines of target responses $\left(\mathrm{Y}_{2}\right.$ and $\left.\mathrm{Y}_{3}\right)$ minimization solutions, evidence the results of the optimization approach A1 for independent minimum values of $Y_{2}$ and $Y_{3}$, and global minimum for both responses. formulation, as predicted by the overall analysis of the process. Overall, the provided analysis shows the advantages of using RSM analysis to understand better complex reactive systems, and the interest to use these statistical approaches as decision-making tools. As future work, the optimized formulations will be tested in the synthesis of rigid polyurethane foams.

\section{Declaration of competing interest}

The authors declare that they have no known competing financial interests or personal relationships that could have appeared to influence the work reported in this paper.

\section{CRediT authorship contribution statement}

João A. Pinto: Conceptualization, Methodology, Investigation, Formal analysis, Writing - original draft. Miguel A. Prieto: Formal analysis, Writing - original draft. Isabel C.F.R. Ferreira: Formal analysis, Resources. Mohamed N. Belgacem: Supervision, Writing - review \& editing. Alírio E. Rodrigues: Supervision, Writing - review \& editing. Maria Filomena Barreiro: Resources, Conceptualization, Supervision, Writing - review \& editing.

\section{Acknowledgements}

Base Funding UIDB/50020/2020 of the Associate Laboratory LSRELCM - funded by national funds through FCT/MCTES (PIDDAC) and base Funding (UIDB/00690/2020) of CIMO - Centro de Investigação de Montanha, funded by national funds through FCT/MCTES (PIDDAC). Project NORTE-01-0145-FEDER-000006, funded by NORTE2020 under PT2020, through FEDER. Financial support of MICINN for the Ramón\& Cajal research grant of M.A. To COST Action LignoCOST (CA17128) supported by COST (European Cooperation in Science and Technology). The authors also thank AMENDOURO for providing the almond shell samples used in this study.

\section{Appendix A. Supplementary data}

Supplementary material related to this article can be found, in the online version, at doi:https://doi.org/10.1016/j.indcrop.2020.112542.

\section{References}

Aktas, T., Thy, P., Williams, R.B., McCaffrey, Z., Khatami, R., Jenkins, B.M., 2015.
Characterization of almond processing residues from the Central Valley of California for thermal conversion. Fuel Process. Technol. 140, 132-147.

Albuquerque, B.R., Prieto, M.A., Barreiro, M.F., Rodrigues, A., Curran, T.P., Barros, L Ferreira, I.C.F.R., 2016. Catechin-based extract optimization obtained from Arbutus unedo L. Fruits using maceration/microwave/ultrasound extraction techniques. Ind. Crops Prod. 95, 404-415.

Aniceto, J.P.S., Portugal, I., Silva, C.M., 2012. Biomass-based polyols through oxypropylation reaction. Chem. Sus. Chem 5, 1358-1368.

Arbenz, A., Avérous, L., 2015. Oxyalkylation of gambier tannin-synthesis and characterization of ensuing biobased polyols. Ind. Crops Prod. 67, 295-304.

ASTM, 2005. American Society for Testing and Materials. Standard Test Method for Testing Polyurethane Raw Materials: Determination of Hydroxyl Numbers of Polyols (D4274). ASTM Standard. ASTM International, West Conshohocken, Philadelphia, USA.

ASTM, 1984. American Society for Testing and Materials. Annual Book of ASTM Standards. ASTM International, West Conshohocken, Philadelphia, USA.

Bernardini, J., Cinelli, P., Anguillesi, I., Coltelli, M.B., Lazzeri, A., 2015. Flexible polyurethane foams green production employing lignin or oxypropylated lignin. Eur. Polym. J. 64, 147-156.

Box, G.E.P., Hunter, J.S., 1957. Multi-factor experimental designs. J. Ann. Math. Stat. 28, 95-241.

Briones, R., Serrano, L., Younes, R., Ben, Mondragon, I., Labidi, J., 2011. Polyol production by chemical modification of date seeds. Ind. Crops Prod. 34, 1035-1040.

Cateto, C.A., Barreiro, M.F., Ottati, C., Lopretti, M., Rodrigues, A.E., Belgacem, M.N., 2013. Lignin-based rigid polyurethane foams with improved biodegradation. J. Cell. Plast. 50, 81-95.

Cateto, C.A., Barreiro, M.M.F., Rodrigues, A.E., Belgacem, M.N., 2009. Optimization study of lignin oxypropylation in view of the preparation of polyurethane rigid foams. Ind. Eng. Chem. Res. 48, 2583-2589.

Comuzzi, C., Polese, P., Melchior, A., Portanova, R., Tolazzi, M., 2003. SOLVERSTAT: a new utility for multipurpose analysis. An application to the investigation of deoxygenated Co(II) complex formation in dimethylsulfoxide solution. Talanta 59, 67-80.

Curran, T.P., Prieto, M.A., Anders, Y., Vázquez, J.A., Murado, M.A., 2013. Development of a bivariate mathematical model to characterize simultaneously the dose-time-responses of pro-oxidant agents. American Society of Agricultural and Biological Engineers Annual International Meeting 2013 4387-4396.

D'Souza, J., George, B., Camargo, R., Yan, N., 2015. Synthesis and characterization of biopolyols through the oxypropylation of bark and alkaline extracts of bark. Ind. Crops Prod. 76, 1-11.

De Lean, A., Munson, P.J., Rodbard, D., 1978. Simultaneous analysis of families of sigmoidal curves: application to bioassay, radioligand assay, and physiological doseresponse curves. Am. J. Physiol. 235, E97-102.

De Menezes, A.J., Pasquini, D., Curvelo, A.A.S., Gandini, A., 2007. Novel thermoplastic materials based on the outer-shell oxypropylation of corn starch granules. Biomacromolecules 8, 2047-2050.

De Rezende, S.C., Pinto, J.A., Fernandes, I.P., Leimann, F.V., Barreiro, M.F., 2018 Oxypropylation of Brazilian pine-fruit shell evaluated by principal component analysis. J. Renew. Mater. 6, 715-723.

Esfahlan, A.J., Jamei, R., Esfahlan, R.J., 2010. The importance of almond (Prunus amygdalus L.) and its by-products. Food Chem. 120, 349-360.

Estevinho, B.N., Ribeiro, E., Alves, A., Santos, L., 2008. A preliminary feasibility study for pentachlorophenol column sorption by almond shell residues. Chem. Eng. J. 136, 188-194.

Evtiouguina, M., Barros-Timmons, A., Cruz-Pinto, J.J., Neto, C.P., Belgacem, M.N., Gandini, A., 2002. Oxypropylation of cork and the use of the ensuing polyols in polyurethane formulations. Biomacromolecules 3, 57-62.

FAOSTAT, 2020. FAOSTAT database on agriculture. FAO - Food Agric. Organ. United 
Nations URL. http://www.fao.org/faostat/en/\#data.

Fernandes, S., Freire, C.S.R., Neto, C.P., Gandini, A., 2007. The bulk oxypropylation of chitin and chitosan and the characterization of the ensuing polyols. Green Chem. 10, 93-97.

Heleno, S.A., Diz, P., Prieto, M.A., Barros, L., Rodrigues, A., Barreiro, M.F., Ferreira, I.C.F.R., 2016. Optimization of ultrasound-assisted extraction to obtain mycosterols from Agaricus bisporus L. by response surface methodology and comparison with conventional Soxhlet extraction. Food Chem. 197, 1054-1063.

Holtman, K.M., Offeman, R.D., Franqui-Villanueva, D., Bayati, A.K., Orts, W.J., 2015. Countercurrent extraction of soluble sugars from almond hulls and assessment of the bioenergy potential. J. Agric. Food Chem. 63, 2490-2498.

INE, 2018. Estatísticas Agrícolas 2018. Instituto Nacional de Estatística, I.P., Lisboa, Portugal.

Intergovernmental Panel on Climate Change (Ed.), 2005. Statistics for Experimenters: Design, Innovation, and Discovery. Cambridge University Press, Cambridge.

Kemmer, G., Keller, S., 2010. Nonlinear least-squares data fitting in Excel spreadsheets. Nat. Protoc. 5, 267-281.

Li, B., Zhou, M., Huo, W., Cai, D., Qin, P., Cao, H., Tan, T., 2020. Fractionation and oxypropylation of corn-stover lignin for the production of biobased rigid polyurethane foam. Ind. Crops Prod. 143, 111887.

Matos, M., Barreiro, M.F., Gandini, A., 2010. Olive stone as a renewable source of biopolyols. Ind. Crops Prod. 7-12.

Murado, M.A., Prieto, M.A., 2013. NOEC and LOEC as merely concessive expedients: two unambiguous alternatives and some criteria to maximize the efficiency of dose-response experimental designs. Sci. Total Environ. 461-462, 576-586.

Nadji, H., Bruzzèse, C., Belgacem, M.N., Benaboura, A., Gandini, A., 2005. Oxypropylation of lignins and preparation of rigid polyurethane foams from the ensuing polyols. Macromol. Mater. Eng. 290, 1009-1016.

Oliveira, F., de, Ramires, E.C., Frollini, E., Belgacem, M.N., 2015. Lignopolyurethanic materials based on oxypropylated sodium lignosulfonate and castor oil blends. Ind. Crops Prod. 72, 77-86.

Pandey, A., Soccol, C.R., Nigam, P., Soccol, V.T., 2000. Biotechnological potential of agroindustrial residues. I: sugarcane bagasse. Bioresour. Technol. 74, 69-80.

Pavier, C., Gandini, A., 2000. Oxypropylation of sugar beet pulp. 1. Optimisation of the reaction. Ind. Crops Prod. 12,1-8.

Prieto, M.A., Vázquez, J.A., 2014. A Time-Dose Model to Quantify the Antioxidant Responses of the Oxidative Hemolysis Inhibition Assay (OxHLIA) and Its Extension to Evaluate Other Hemolytic Effectors. Biomed Res. Int. 201415 pages.

Rosa, J.R., da Silva, I.S.V., de Lima, C.S.M., Flauzino Neto, W.P., Silvério, H.A., dos Santos, D.B., Barud, HdaS., Ribeiro, S.J.L., Pasquini, D., 2014. New biphasic monocomponent composite material obtained by partial oxypropylation of bacterial cellulose. Cellulose 21, 1361-1368.

Rosa, J.R., da Silva, I.S.V., de Lima, C.S.M., Pasquini, D., 2015. Production of polyols and new biphasic mono-component materials from soy hulls by oxypropylation. Ind Crops Prod. 72, 152-158.

Serrano, L., Alriols, M.G.M., Briones, R., Mondragon, I., Labidi, J., 2010. Oxypropylation of rapeseed cake residue generated in the biodiesel production process. Ind. Eng. Chem. Res. 49, 1526-1529.

Shi, P., Tsai, C.-L., 2002. Regression model selection: a residual likelihood approach. J. R. Stat. Soc. B (Statistical Methodol. 64, 237-252.

TAPPI, 2007. T 211 om-07, ash in Wood pulp, paper and paper- board. TAPPI Test Methods. PAPPI Press, Atlanta.

TAPPI, 2006. T 222 om-08 and acid-insoluble lignin in Wood and pulp. TAPPI Test Methods. APPI Press, Atlanta.

Valdés, A., Vidal, L., Beltrán, A., Canals, A., Garrigós, M.C., 2015. Microwave-assisted extraction of phenolic compounds from almond skin byproducts (Prunus amygdalus): a multivariate analysis approach. J. Agric. Food Chem. 63, 5395-5402. 